\title{
Image Watermark Based on Extended Shearlet and Insertion Using the Largest Information Entropy on Horizontal Cone
}

\author{
Zhao Jian, ${ }^{1}$ Sun Meiling, ${ }^{1}$ Jia Jian, ${ }^{2}$ Huang Luxi, ${ }^{1}$ Han Fan, ${ }^{1}$ and Liu Shan ${ }^{1}$ \\ ${ }^{1}$ School of Information Science and Technology, Northwest University, Xi'an 710069, China \\ ${ }^{2}$ School of Mathematical Science, Northwest University, Xian 710069, China
}

Correspondence should be addressed to Zhao Jian; zjctec@nwu.edu.cn

Received 24 March 2015; Accepted 17 May 2015

Academic Editor: Chih-Cheng Hung

Copyright (C) 2015 Zhao Jian et al. This is an open access article distributed under the Creative Commons Attribution License, which permits unrestricted use, distribution, and reproduction in any medium, provided the original work is properly cited.

\begin{abstract}
Extended discrete shearlet provides a directional multiresolution decomposition. It has been mathematically shown that extended discrete shearlet is a more efficient representation for the signals containing distributed discontinuities such as edges, compared to discrete wavelet. Multiresolution analyses such as curvelet and ridgelet share similar properties, yet their directional representations are significantly different from that of extended discrete shearlet. Taking advantage of the unique properties of directional representation of extended discrete shearlet, we develop an image watermark algorithm based on the largest information entropy. In proposed algorithm, firstly, 1-level extended discrete shearlet transform decomposes the test image into directional components on horizontal cone; each directional component reflects directional features and textured features differently. Next, the directional component whose information entropy is the highest is selected to carry watermark. Compared with related algorithms based on DWT and DCT, the proposed algorithm tends to obtain preferable invisibility when it is robust against common attacks.
\end{abstract}

\section{Introduction}

Image watermark is one of the most active and challenging subjects in the information hiding research because it is an efficient solution to protect the copyright of the digital media. Wavelets have been widely applied in image watermark owning to their perfect performance for piecewise smooth signals in one dimension $[1,2]$.

However, wavelets are not actually optimal representation for signals in two dimensions or higher dimensions such as images [3-7]. In fact, wavelets not only fail to preserve the detailed features but they also fail to capture the directional features $[3,7]$. With the development of multiresolution analysis, various multiresolution methods have been proposed to overcome this limitation of wavelets, and it has been experimentally proved that these multiresolution methods perform well in image watermark $[4,5,8]$. Among various multiresolution methods, discrete shearlet $[6,9]$ is a relatively new multiresolution method, which has drawn considerable attention and has been applied in some fields of image processing such as image denoising, image approximations, and image inpainting for its superior performance in dealing with signals in high dimensions [6,9-11]. Extended discrete shearlet $[9,11]$ is derived from discrete shearlet transform. It is based on a simple but rigorous mathematical framework which is composed of directional orthonormal bases; each orthonormal base exhibits high directional sensitivity and is highly anisotropic. The extended discrete shearlet transform attempts to obtain a sparse image representation which contains more directional information and preserves more detailed information than discrete wavelet.

Taking advantage of these unique properties of extended discrete shearlet, we develop an image watermark algorithm based on extended discrete shearlet transform and the largest information entropy $[12,13]$. In proposed algorithm, by using extended discrete shearlet transform, an image is composed into directional components on the horizontal cone; each directional component is transformed into a set of shearlet coefficients. The watermark is embedded into the largest coefficients of the directional component whose information entropy is the highest. The proposed algorithm is compared with DCT-based algorithm and DWT-based algorithm. 


\section{Correlation with Basic Theory}

2.1. Correlation with Shearlet Transform. A 2D affine system with composite dilations $[6,9,14]$ is described by

$$
\begin{aligned}
& \mathrm{SH}(\psi)=\left\{\psi_{a, s, t}(x)=a^{-3 / 4} \psi\left(A_{a}^{-1} B_{s}^{-1}(x-t)\right)\right. \\
& =a^{-3 / 4} \psi\left(\left(\begin{array}{cc}
\frac{1}{a} & -\frac{s}{a} \\
0 & \frac{1}{\sqrt{a}}
\end{array}\right) x-t\right), a>0, s \in R, t \\
& \left.\in R^{2}\right\}
\end{aligned}
$$

where the parabolic scaling matrices $A_{a}=(a, 0 ; 0, \sqrt{a})(a>$ $0)$ are associated with scale transform and the shear matrices $B_{s}=(1, s ; 0,1)$ are related to area-preserving geometrical transform. The generating function $\psi$ is chosen like a tensor product by

$$
\widehat{\psi}(\xi)=\widehat{\psi}\left(\xi_{1}, \xi_{2}\right)=\widehat{\psi}_{1}\left(\xi_{1}\right) \widehat{\psi}_{2}\left(\frac{\xi_{2}}{\xi_{1}}\right),
$$

where $\psi_{1}$ is continuous wavelet which is supported with supp $\widehat{\psi}_{1} \subset[-2,-1 / 2] \cup[1 / 2,2]$ and $\psi_{2}$ is a bump function with certain weak additional properties and is supported with supp $\widehat{\psi}_{2} \subset[-1,1]$.

The associated continuous shearlet transform $[6,9-11,14]$ is defined by

$$
\mathrm{SH}_{f}(a, s, t)=\left\langle f, \psi_{a, s, t}\right\rangle .
$$

The shearlet transform is a function associated with $a, s$, and $t$, whereas wavelet transform is a function which is merely associated with $a$ and $t$. The main idea of the shear parameter $s$ is to capture directional features, such as orientations of curves $[6,9,11]$. As a consequence, the shearlet is able to capture directional information. Figure 1 illustrates the ability of capturing directions of the shearlet transform.

2.2. Correlation with Discrete Shearlet Transform. By sampling $\mathrm{SH}(\psi)$ on an appropriate discrete set of $a, s$, and $t$, a discrete shearlet transform is obtained $[6,9,10,14]$. The discrete shearlet transform is described by

$$
\begin{gathered}
\left\{\psi_{j, l, k}^{(d)}(x)=\left|\operatorname{det} A_{d}\right|^{j / 2} \psi\left(B_{d}^{l} A_{d}^{j} x-k\right): j \geq 0,\right. \\
\left.-\left\lceil 2^{j / 2}\right\rceil \leq l \leq\left\lceil 2^{j / 2}\right\rceil, k \in Z^{2}, d=0,1\right\},
\end{gathered}
$$

where $A_{0}=(4,0 ; 0,2), A_{1}=(2,0 ; 0,4), B_{0}=(1,0 ; 1,1)$, and $B_{1}=(1,1 ; 0,1)$. If $\psi_{j, l, k}^{(d)}$ satisfy the property

$$
\sum_{j, l, k}\left|\left\langle f, \psi_{j, l, k}^{(d)}\right\rangle\right|^{2}=\|f\|^{2}
$$

the discrete shearlet transform forms a tight frame for $L^{2}(R)$.
For any $\xi=\left(\xi_{1}, \xi_{2}\right) \in \widehat{R}^{2}, \xi_{1} \neq 0$, suppose

$$
\begin{gathered}
\sum_{j \geq 0}\left|\widehat{\psi}_{1}\left(2^{-2 j} \omega\right)\right|^{2}=1 \quad \text { for }|\omega| \geq \frac{1}{8} \\
\sum_{l=-2^{j}}^{2^{j}}\left|\widehat{\psi}_{2}\left(2^{j} \omega-l\right)\right|^{2}=1 \quad \text { for }|\omega| \leq 1 .
\end{gathered}
$$

Specifically, define

$$
\begin{aligned}
& \widehat{\psi}^{(0)}(\omega)=\widehat{\psi}^{(0)}\left(\omega_{1}, \omega_{2}\right)=\widehat{\psi}_{1}\left(\omega_{1}\right) \widehat{\psi}_{2}\left(\frac{\omega_{2}}{\omega_{1}}\right), \\
& \widehat{\psi}^{(1)}(\omega)=\widehat{\psi}^{(1)}\left(\omega_{1}, \omega_{2}\right)=\widehat{\psi}_{1}\left(\omega_{2}\right) \widehat{\psi}_{2}\left(\frac{\omega_{1}}{\omega_{2}}\right),
\end{aligned}
$$

where $\psi_{1}$ is a discrete wavelet function with supp $\widehat{\psi}_{1} \subset$ $[-1 / 2,-1 / 16] \cup[1 / 16,1 / 2]$ and $\psi_{2}$ is a bump function with supp $\widehat{\psi}_{2} \subset[-1,1]$. From the supporting condition of $\widehat{\psi}_{1}$ and $\widehat{\psi}_{2}, \psi_{j, l, k}$ in frequency domain is supported with

$$
\begin{gathered}
\operatorname{supp} \widehat{\psi}_{j, l, k}^{(0)} \subset\left\{\left(\omega_{1}, \omega_{2}\right): \omega_{1} \in\left[-2^{2 j-1},-2^{2 j-4}\right]\right. \\
\left.\cup\left[2^{2 j-4}, 2^{2 j-1}\right],\left|l 2^{-j}+\frac{\omega_{2}}{\omega_{1}}\right| \leq 2^{-j}\right\} .
\end{gathered}
$$

Equation (8) implies that each element $\widehat{\psi}_{j, l, k}$ is supported on a pair of trapezoids, each of which is contained in a box of approximate size $2^{2 j} \times 2^{j}$ and oriented along line of slope $-l 2^{-j}[6,9,10,14]$.

2.3. Correlation with Extended Discrete Shearlet. Extended discrete shearlet $[6,11]$ is obtained by directly extending discrete shearlet. Yet its framework is slightly different from that of the discrete shearlet. Framework of the extended discrete shearlet is composed of directional orthonormal bases; each orthonormal basis provides anisotropic window functions elongated along the direction determined by shear matrix. In addition, the extended discrete shearlet framework uses a MRA approach to compute shearlet coefficients and uses an iterative method to approximate the inverse. Based on this construction, a given image is explicitly decomposed into variously directional components for different cones when extended discrete shearlet is associated with different shear matrices and direction numbers; each directional component reflects directional features and textured features differently (see Figure 2); besides, a stable reconstruction of a given image is obtained.

Actually, the extended discrete shearlet framework exhibits highly directional sensitivity; and, furthermore, the extended discrete shearlet framework is optimally sparse representation and is well localized in both the spatial and the frequency domains. Based on these properties, the extended discrete shearlet can be considered as an appropriate transform for image watermark. 


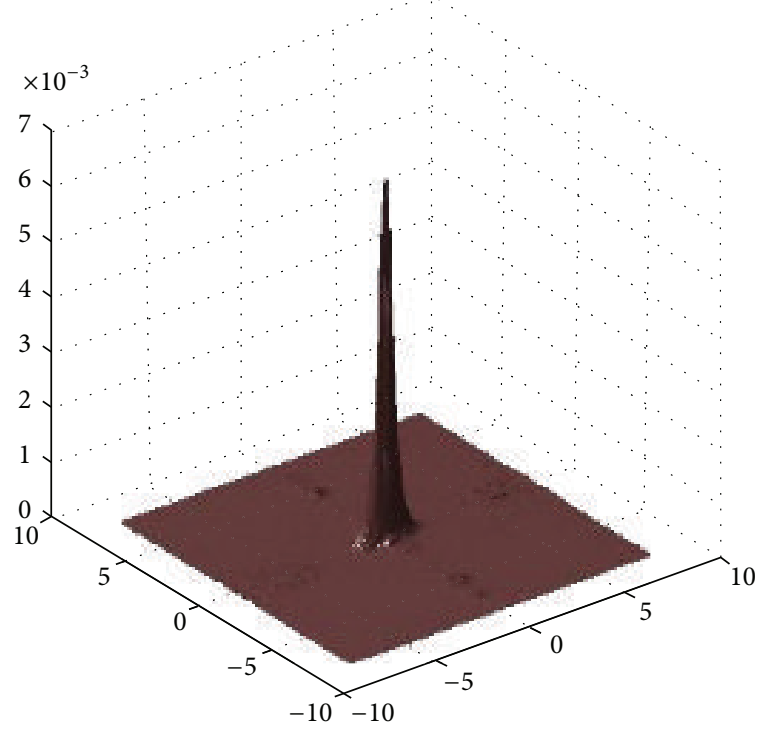

(a)

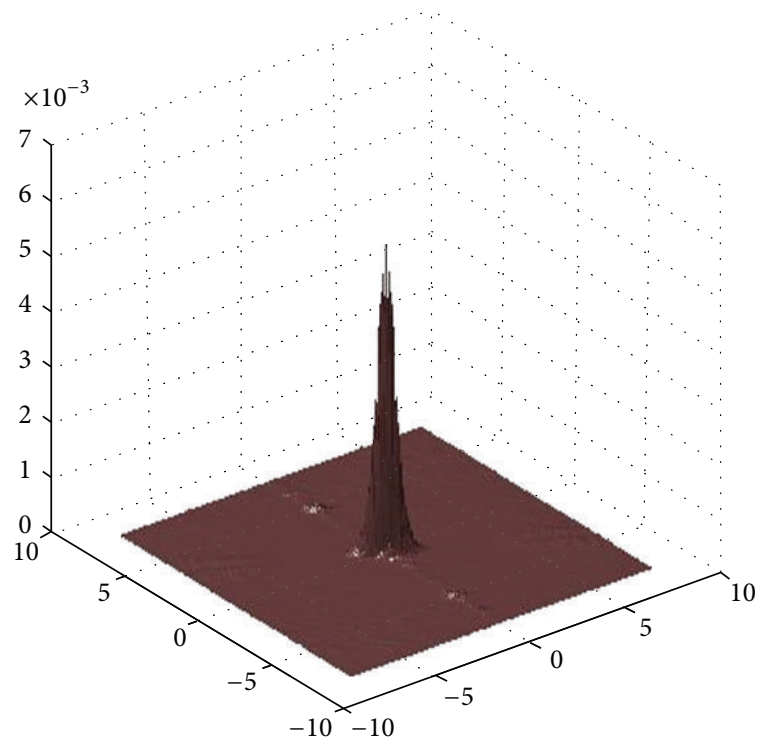

(c)

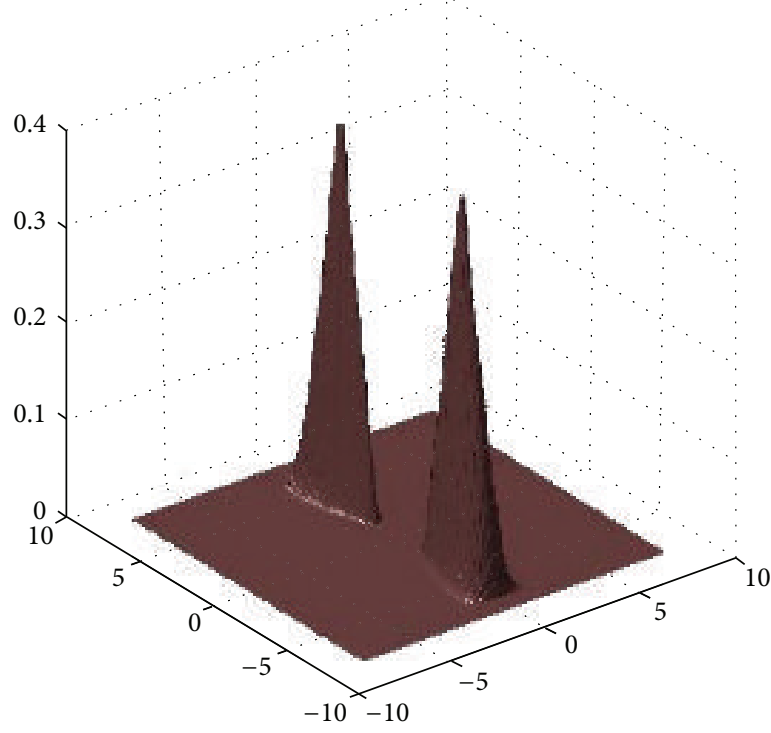

(b)

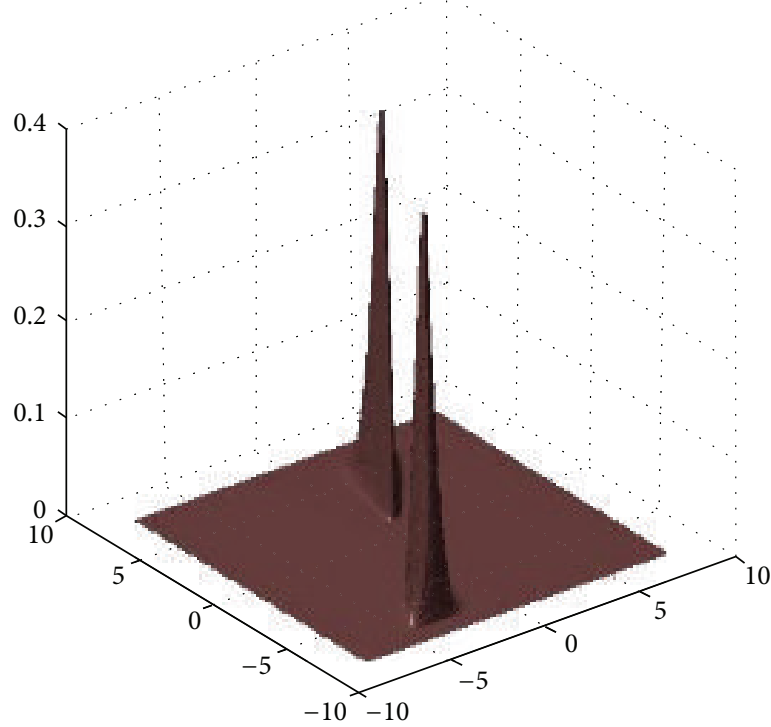

(d)

FIGURE 1: (a) $\psi_{a, s, t}$ in the time domain for $a=0.3, s=0$, and $t=0$. (b) $\psi_{a, s, t}$ in the frequency domain for $a=0.3, s=0$, and $t=0$. (c) $\psi_{a, s, t}$ in the time domain for $a=0.3, s=-0.5$, and $t=0$. (d) $\psi_{a, s, t}$ in frequency domain for $a=0.3, s=-0.5$, and $t=0$.

\section{A Novel Watermark Algorithm Based on Extended Discrete Shearlet}

The most important issue in extended discrete shearletbased watermark algorithm is how to choose the watermark embedding location or the effective coefficients to be embedded, since the choice of watermark embedding location has an effect on both invisibility and robustness of watermark algorithm to some extent $[1,2,4,5,8]$. In this section, a novel algorithm for image watermark is proposed, which is based on combining the analysis of extended discrete shearlet coefficients $[6,11]$ and the basic theory of the largest information entropy to search the watermark embedding location for different images.
3.1. Analysis of Extended Discrete Shearlet Coefficients. After 1-level extended discrete shearlet decomposition associated with shear matrix $B_{0}$ and direction numbers $m$, the test image of size $N \times N$ is decomposed into $m$ directional components on horizontal cone (see Figure 2(b)); each of directional components is transformed into a set of shearlet coefficients.

Each directional component reflects directional features and preserved textured features differently; therefore, the occurring probabilities of different pixel values in each directional component are different from those of all others and the probability distribution of each directional component's information is different from that of all others in confusion degree. The higher information entropy $[12,13]$ of the directional component is, the more uncertain occurring 


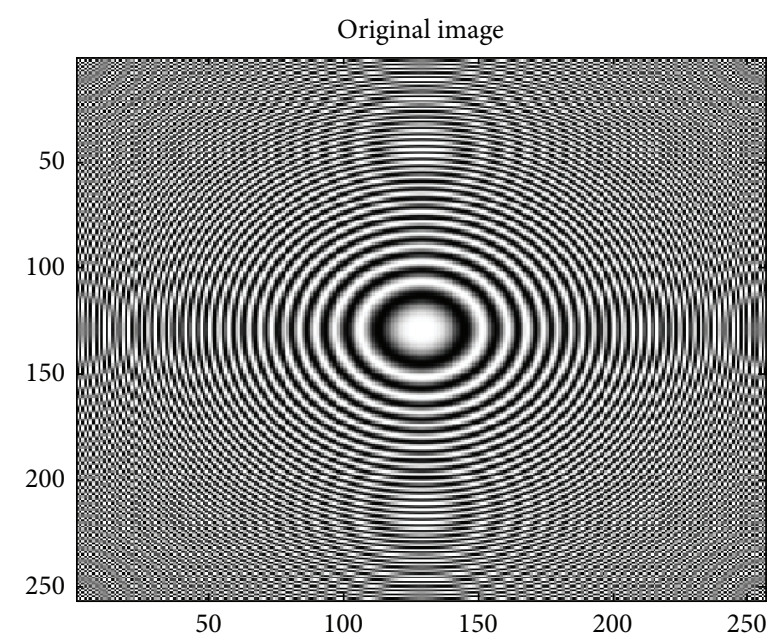

(a)
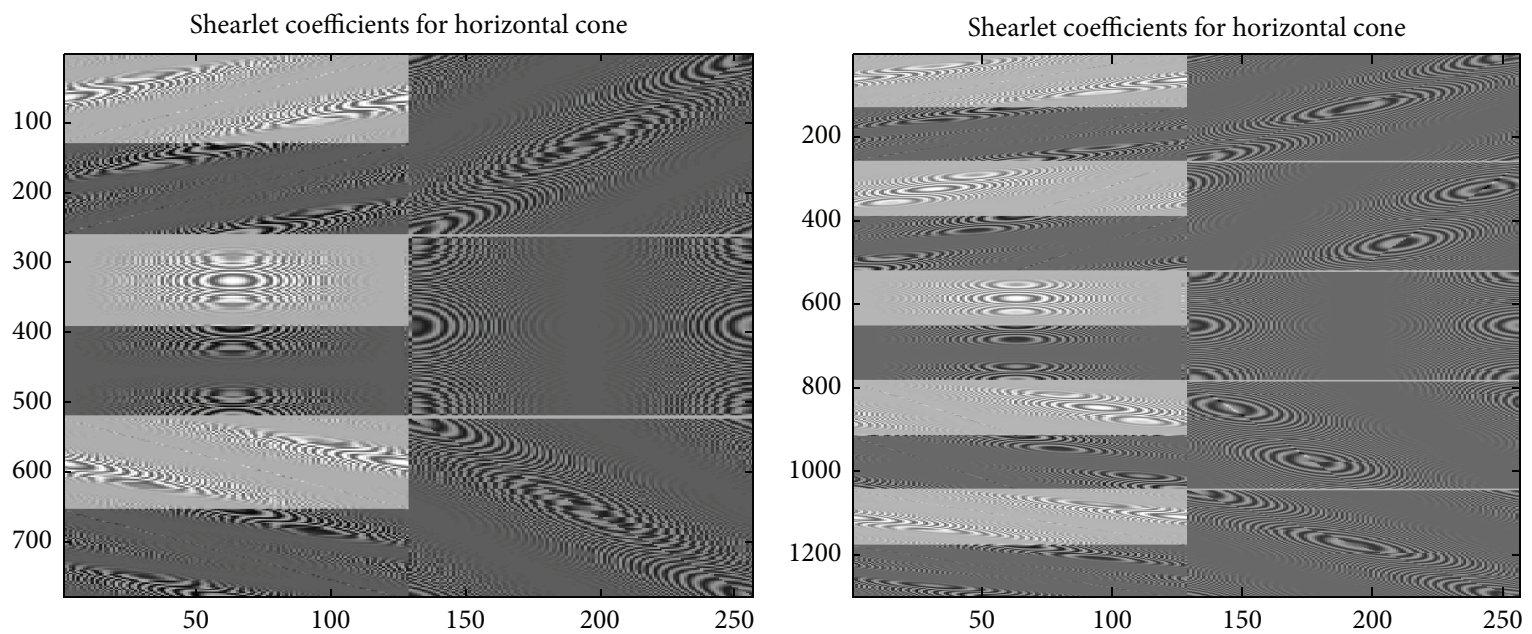

(b)
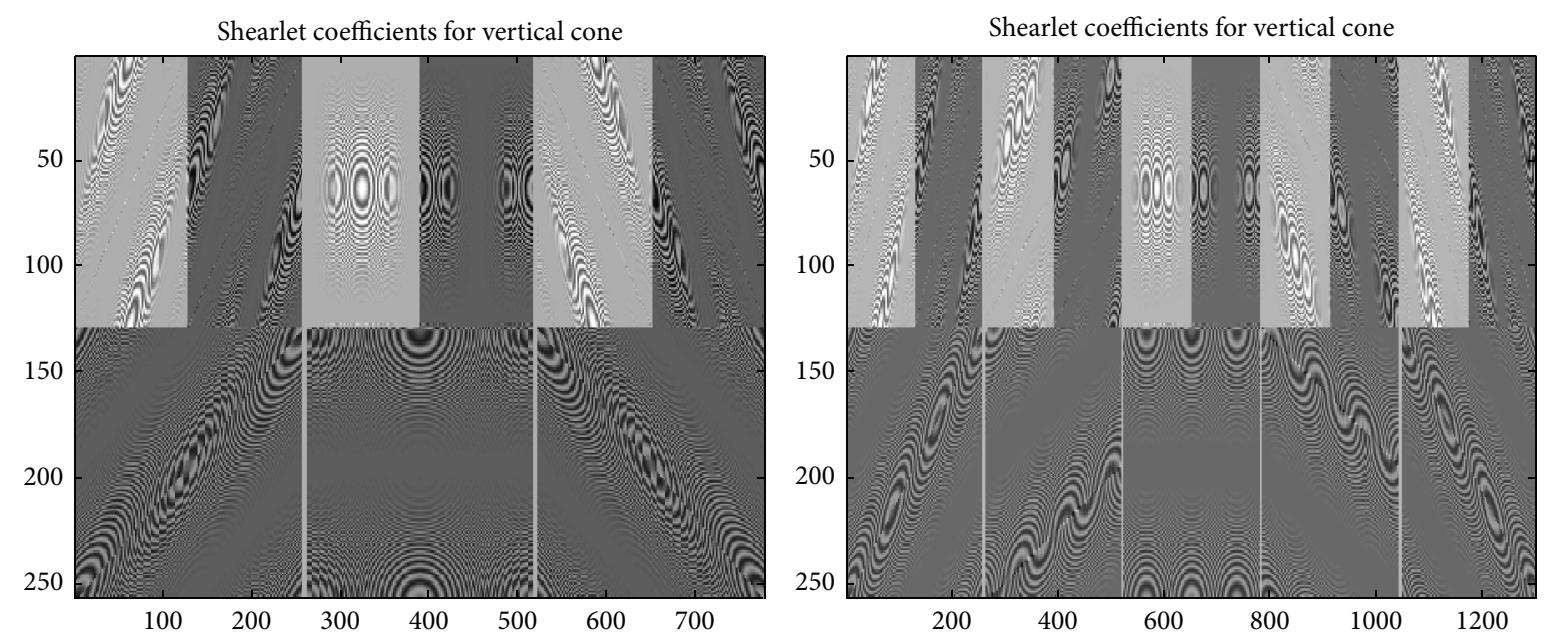

(c)

FIGURE 2: The extended discrete shearlet transform results corresponding to different directions and shear matrices. 
probabilities of its different pixel values are, and probability distribution of its information is more disordered and more chaotic; that means it can hide more watermark bits. Based on this analysis, the directional component whose information entropy is the largest is selected to carry watermark. The information entropy of the $l$ th directional component on horizontal cone is computed as follows:

(1) Mark pixel values of the $l$ th directional component by $\left(X_{1}^{l}, X_{2}^{l}, \ldots, X_{N * N}^{l}\right)$.

(2) Count occurring probability $\left(p_{1}^{l}, p_{2}^{l}, \ldots, p_{j}^{l}, \ldots, p_{K}^{l}\right)$ of different pixel values of the $l$ th directional component; here $K$ is the total number of different pixel values.

(3) Calculate the information entropy of the $l$ th directional component by

$$
S^{l}=-\sum_{j=1}^{K} p_{j}^{l} \log _{2}\left(p_{j}^{l}\right)
$$

where $l(l=1, \ldots, m)$.

Generally, watermark insertion is realized by modifying some special values of pixels or transforming domain coefficients $[1,2,4,5]$. Here watermark insertion is realized by modifying the largest $n \times n$ coefficients of selected directional component in extended discrete shearlet domain.

\subsection{Watermark Insertion Using the Largest Information} Entropy. For an image $w$ used as watermark, in order to uniformly and randomly spread bits over the host image, it needs to be transformed into a binary image by

$$
w w(i, j)= \begin{cases}-1 & \text { if } w(i, j)>\operatorname{Tr} \\ 1 \quad \text { else, } & 1 \leq i, j \leq n\end{cases}
$$

Here threshold Tr is selected by OSTA method. After processing the watermark image $w$, a binary image $w w$ is obtained and then reshaped to binary sequence $w m$ with length $n \times n$.

According to the above analysis in Section 3.1, the directional component, whose information entropy is the highest, can hide more watermark bits and is selected to carry watermark. To obtain directional components on horizontal cone, a l-level extended discrete shearlet decomposition is applied to test image $I$ with size $N \times N$, which is associated with shear matrix $B_{0}$ and direction numbers $m$. Then, to find out the directional component whose information entropy is the highest, information entropy of each directional component on horizontal cone is computed by (9).

Here we suppose the $p$ th $(1 \leq p \leq m)$ directional component's information entropy is the highest. Then the binary sequence $w m$ is embedded into the largest $n \times n$ coefficients of the $p$ th directional component by

$$
\begin{aligned}
& y^{(p)}(i, j) \\
& \quad= \begin{cases}x^{(p)}(i, j)(1+a \times b(k)), & \text { if } x^{(p)}(i, j) \geq T \\
x^{(p)}(i, j), & \text { else, }\end{cases}
\end{aligned}
$$

where $1 \leq k \leq n \times n, a$ is used to control embedding strength, and $T$ is the threshold.

Finally, the watermarked image $I_{w}$ is obtained after 1-level extended inverse discrete shearlet transform by using these modified shearlet coefficients.

3.3. Watermark Extraction. The watermark can be extracted correctly from the watermarked image without original test image. The extracting process is as follows, which is mirroring embedding process.

To extract embedded watermark bits from the watermarked image, 1-level extended discrete shearlet decomposition is firstly applied to the watermarked image, where the shear matrix is selected as $B_{0}$ and the number of direction is $n$; then shearlet coefficients of every directional component on horizontal cone are obtained.

Then, the watermarked coefficients are found out according to watermark size and the location key of embedding watermark. The extracted watermark bits are recovered into a sequence $\vec{m}$ by

$$
m(t)=\frac{\left(x_{i j}^{\prime(p)}-x_{(i, j)}^{(p)}\right)}{a \times x_{(i, j)}^{(p)}}, \quad \text { when } x_{i j}^{(p)} \geq T,
$$

where $1 \leq i, j \leq N, 1 \leq t \leq n \times n$, and $T$ is the threshold.

Finally, the extracted watermark $w w^{\prime}$ is recovered by reshaping $\vec{m}$.

\section{Experimental Results}

4.1. Invisibility of Watermark. Invisibility is an evaluative measure of perceptual quality of the watermarked image. In a satisfactory image watermark algorithm, watermark should not cause much degradation of perceptual quality of the watermarked image. In the proposed algorithm, a $64 \times 64$ watermark image is embedded into different test images ("Lena," "Boat," and "Barbara" with $512 \times 512$ ) to test invisibility, where the number of directions is 9 and the embedding strength is 0.1 .

As shown in Table 1, there are not much visual differences between original test images and their corresponding watermarked images. In addition, the PSNR value of each watermarked image is above $53 \mathrm{~dB}$, and NC value of corresponding extracted watermark image is above 0.98 . The extracted watermarks are all easily distinguishable. Whether from a subjective view or from the objective view, the proposed algorithm has preferable invisibility.

Furthermore, by analyzing the absolute difference between the test image and the watermarked image, it is not difficult to find that the watermark tends to spread on these regions with strong edges and extreme brightness. In particular, it is noted that the watermarked image is magnified a hundred times.

4.2. Robustness of the Proposed Algorithm. Robustness is a default measure which is used to evaluate the watermarked image's resistance against attacks. In a satisfactory algorithm for image watermark, the watermark would not be easily 
TABLE 1: The experimental results of invisibility.

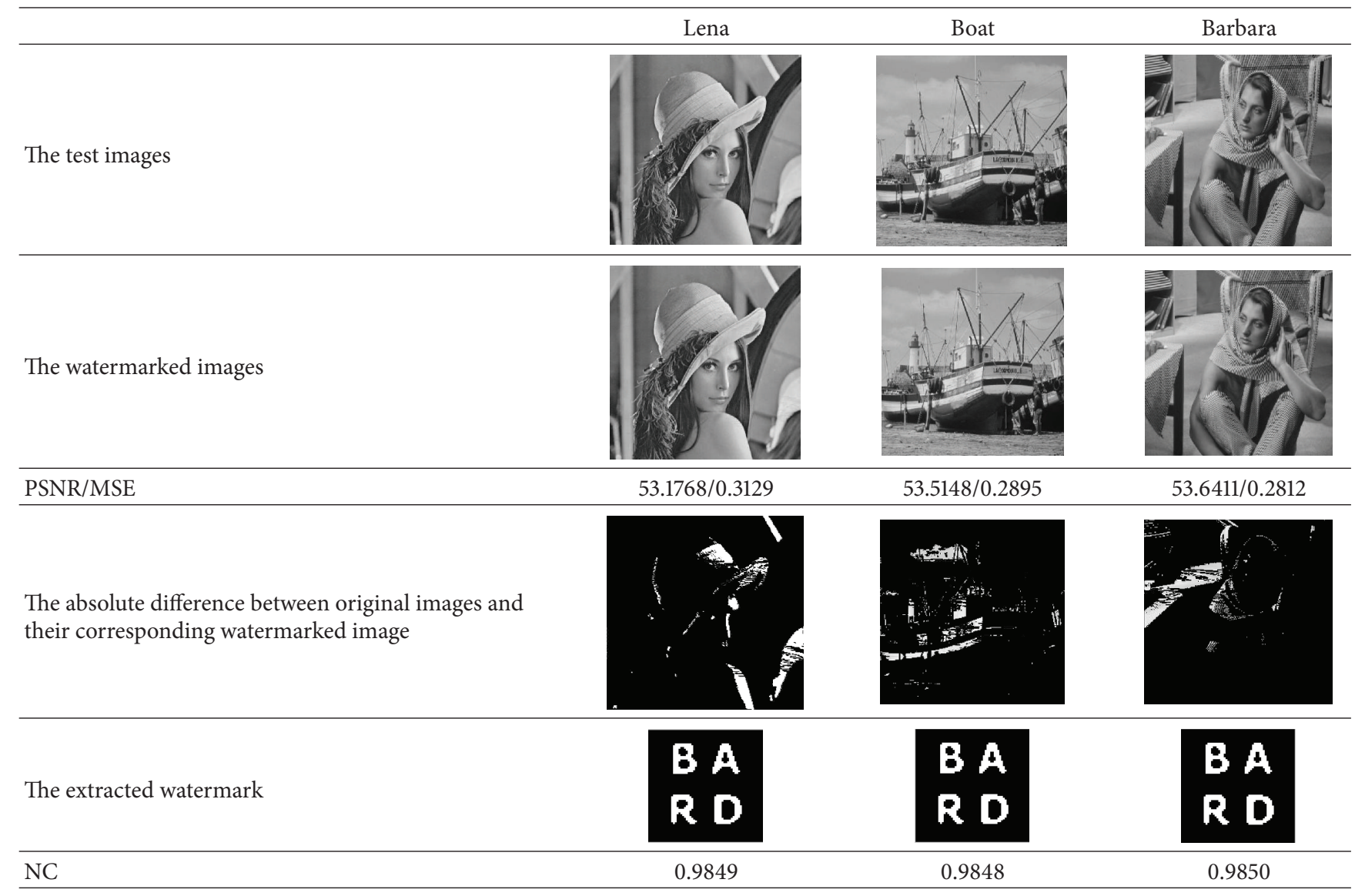

removed from the watermarked image after common and deliberate attacks. In this paper, we simulate the real situation of disguised attacks and check out robustness for the proposed algorithm, where the number of orientations is 9 and the embedding strength is 0.1 .

4.2.1. Robustness to Scaling Attack. For the "Lena" image and "Barbara" image, when scaling parameters are 1/2, 3/4, 3/2, 2 , and 4 , the extracted watermarks are still distinguishable and NC values are all above 0.90 . For the "Boat" image, the extracted watermarks are still distinguishable and $\mathrm{NC}$ values are all above 0.90 when scaling parameters are $3 / 4,3 / 2,2$, and 4 . With the increase of scaling attack parameter, PSNR values are increasing; MSE values are decreasing. Therefore, the proposed algorithm can resist scaling attack.

4.2.2. Robustness to JPEG Compression Attack. JPEG lossy compression is the most common attack during the transformation of the watermarked image. In order to evaluate robustness of proposed algorithm, every watermarked image is compressed using JPEG with different quality factors. From extracted watermarks in Table 3 , it is not hard for anyone to observe that extracted watermark is still distinguishable until the quality factor is set to 30 . In addition, for all test images, PSNR values are all above $30 \mathrm{~dB}$; NC values are all above 0.8 . Therefore, the proposed algorithm is very robust against JPEG compression.
4.2.3. Robustness to Filtering Attack. As shown in Table 4, whether Gaussian low-pass filtering, average filtering, or median filtering, the extracted watermarks are still distinguishable for the "Lena" image and the "Barbara" image. In addition, NC values are all above 0.855 for the "Lena" image, 0.77 for the "Boat" image, and 0.86 for the "Barbara" image. However, the extracted watermarks are imperfect for the "Boat" image.

4.2.4. Robustness under Various Noises Attack. The watermarked image is easily disturbed by various noises. In the test, we add "Salt and Pepper" (Table 5) noise and "Gaussian" white noise (Table 6) to the watermarked images.

For the watermarked image with "Salt and Pepper" noise, with the increase of noise attack strength, more and more noise points appear in the extracted watermark, but the extracted watermark is still distinguishable and complete until the attack strength is set to 0.05 . When the attack strength is set to 0.05 , the extracted watermark is not perfect but distinguishable; the NC value is 0.8262 for the Lena image, 0.7866 for the Boat image, and 0.7861 for the Barbara image, respectively.

Similarly, for the watermarked image under the attack of "Gaussian" white noise, when the noise mean is set to zero, noise points increasingly appear in extracted watermark with the increase of noise variance. The extracted watermarks are 
TABLE 2: Experimental results under the scaling attack.

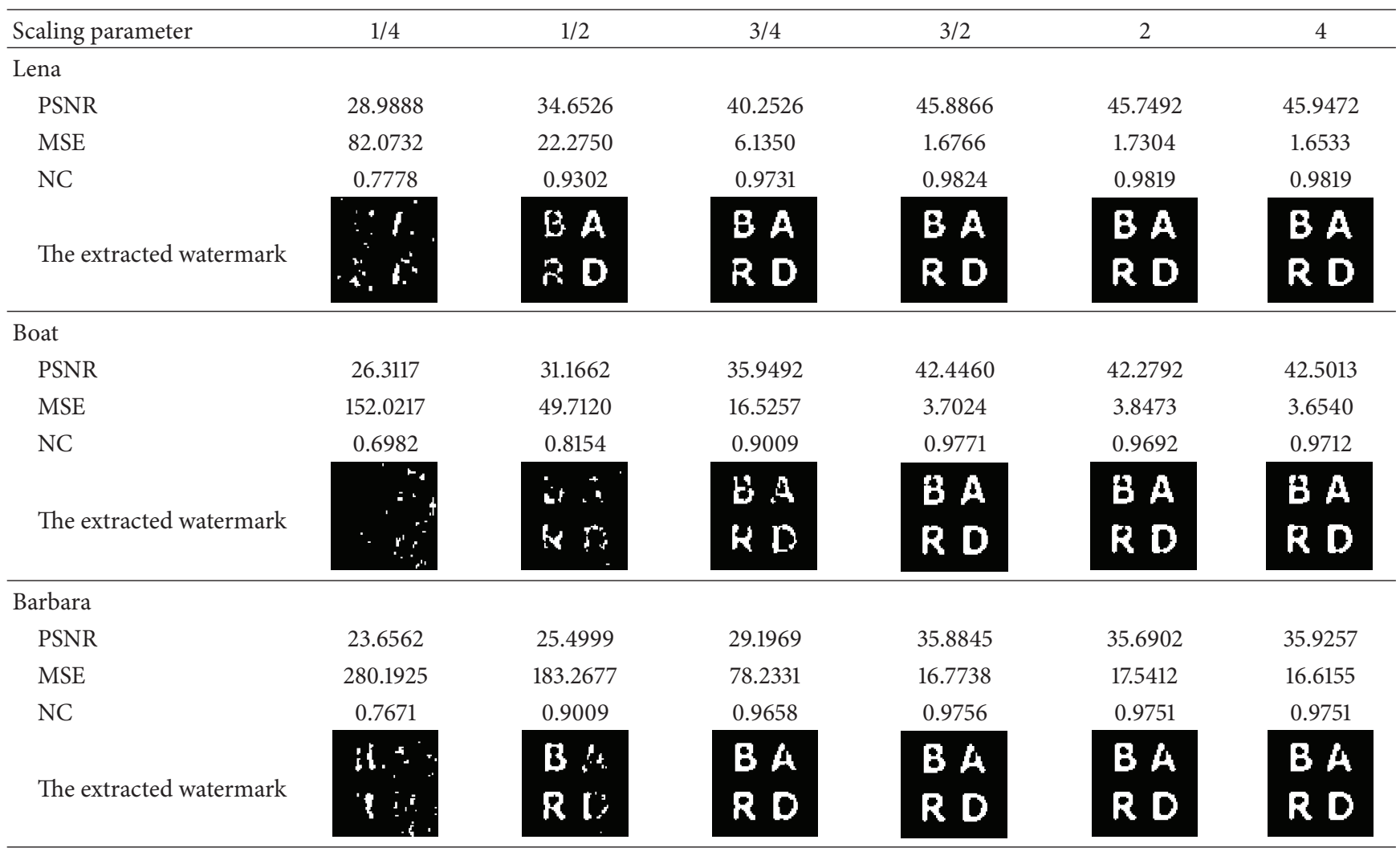

TABLE 3: Experimental results under the attack of JPEG lossy Compression.

\begin{tabular}{|c|c|c|c|c|c|c|}
\hline Quality factor & 30 & 40 & 50 & 60 & 75 & 90 \\
\hline \multicolumn{7}{|l|}{ Lena } \\
\hline PSNR & 35.1718 & 36.0294 & 36.4005 & 42.2603 & 53.2168 & 48.0330 \\
\hline MSE & 19.7649 & 16.0294 & 14.8947 & 6.1242 & 0.3100 & 0.8313 \\
\hline NC & 0.8252 & 0.8506 & 0.9004 & 0.9160 & 0.9453 & 0.9849 \\
\hline \multicolumn{7}{|l|}{ The extracted watermark } \\
\hline \multicolumn{7}{|l|}{ Boat } \\
\hline PSNR & 33.3926 & 34.3324 & 34.6845 & 38.6331 & 53.5308 & 47.9809 \\
\hline MSE & 29.7727 & 23.9794 & 22.1121 & 8.9077 & 0.2884 & 1.0351 \\
\hline $\mathrm{NC}$ & 0.8101 & 0.8423 & 0.8955 & 0.9077 & 0.9233 & 0.9834 \\
\hline The extracted watermark & is & $\begin{array}{l}i t \\
t i\end{array}$ & $\begin{array}{l}9 A \\
\Rightarrow\end{array}$ & $\begin{array}{l}3 A \\
012\end{array}$ & $\begin{array}{l}b A \\
\theta\end{array}$ & $\theta 0$ \\
\hline \multicolumn{7}{|l|}{ Barbara } \\
\hline PSNR & 30.6849 & 31.9392 & 32.2378 & 36.1950 & 53.8219 & 46.1332 \\
\hline MSE & 55.5383 & 41.6065 & 38.8416 & 15.6165 & 0.2697 & 1.5840 \\
\hline $\mathrm{NC}$ & 0.8257 & 0.8457 & 0.8706 & 0.9146 & 0.9438 & 0.9834 \\
\hline & $p_{*} \cdot t_{i}^{4}$ & $4 n$ & $13 A$ & B A & $B / 4$ & $8 A$ \\
\hline The extracted watermark & & $t i$ & & & $\mathrm{RH}$ & \\
\hline
\end{tabular}


TABLE 4: Experimental results under filtering attack.

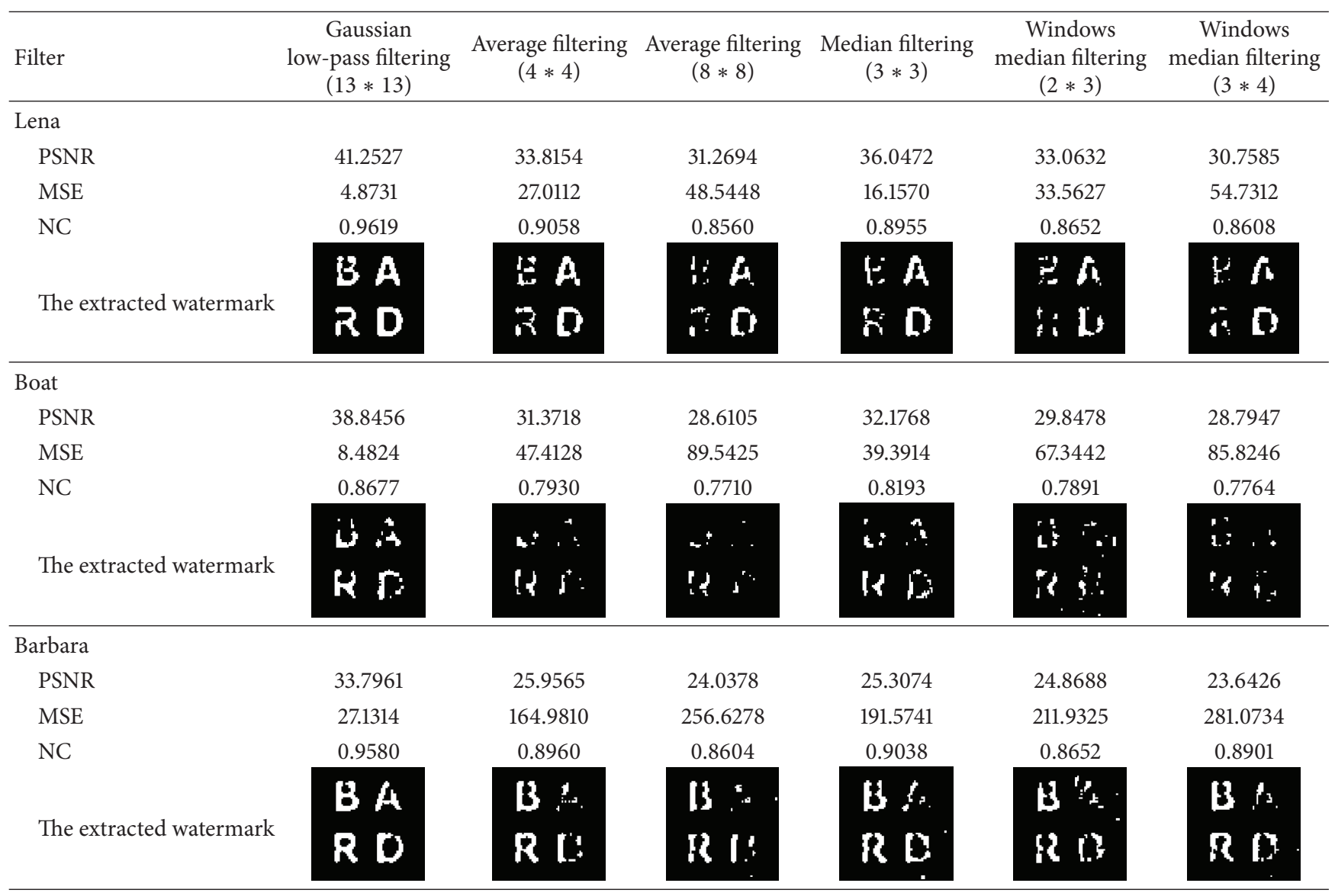

TABLE 5: Experimental results under the attack of "Salt and Pepper" noise.

\begin{tabular}{|c|c|c|c|c|c|}
\hline Attack strength & $d=0.001$ & $d=0.005$ & $d=0.01$ & $d=0.02$ & $d=0.05$ \\
\hline \multicolumn{6}{|l|}{ Lena } \\
\hline PSNR & 34.8891 & 28.3823 & 25.3281 & 22.3135 & 18.4735 \\
\hline MSE & 21.0945 & 94.3727 & 195.0891 & 381.7102 & 924.1257 \\
\hline NC & 0.9814 & 0.9741 & 0.9741 & 0.9268 & 0.8262 \\
\hline \multicolumn{6}{|l|}{ The extracted watermark } \\
\hline \multicolumn{6}{|l|}{ Boat } \\
\hline PSNR & 35.5080 & 28.3722 & 25.3806 & 22.4128 & 18.3500 \\
\hline MSE & 18.2927 & 94.5926 & 188.3749 & 373.0794 & 950.7809 \\
\hline NC & 0.9834 & 0.9712 & 0.9482 & 0.9331 & 0.7866 \\
\hline \multicolumn{6}{|l|}{ The extracted watermark } \\
\hline \multicolumn{6}{|l|}{ Barbara } \\
\hline PSNR & 35.3764 & 28.0243 & 25.2388 & 22.5369 & 18.4612 \\
\hline MSE & 18.8555 & 102.4817 & 194.6269 & 362.5728 & 926.4612 \\
\hline $\mathrm{NC}$ & 0.9814 & 0.9717 & 0.9727 & 0.9316 & 0.7861 \\
\hline The extracted watermark & & & & & \\
\hline
\end{tabular}


TABLE 6: Experimental results under the attack of "Gaussian" white noise $($ mean $=0)$.

\begin{tabular}{|c|c|c|c|c|c|}
\hline Variance & $v=0.00001$ & $v=0.00005$ & $v=0.0001$ & $v=0.0005$ & $v=0.001$ \\
\hline \multicolumn{6}{|l|}{ Lena } \\
\hline PSNR & 47.9293 & 42.5216 & 39.7561 & 32.9411 & 29.9570 \\
\hline MSE & 1.0475 & 3.6585 & 6.8782 & 33.0349 & 65.6718 \\
\hline $\mathrm{NC}$ & 0.9849 & 0.9766 & 0.9575 & 0.8721 & 0.8345 \\
\hline \multicolumn{6}{|l|}{ The extracted watermark } \\
\hline \multicolumn{6}{|l|}{ Boat } \\
\hline PSNR & 48.0248 & 42.5474 & 39.7670 & 32.9706 & 29.9739 \\
\hline MSE & 1.0247 & 3.6169 & 6.8608 & 32.8114 & 65.4169 \\
\hline $\mathrm{NC}$ & 0.9854 & 0.9697 & 0.9565 & 0.8643 & 0.8179 \\
\hline \multicolumn{6}{|l|}{ The extracted watermark } \\
\hline \multicolumn{6}{|l|}{ Barbara } \\
\hline PSNR & 48.0677 & 42.5511 & 39.7595 & 32.9428 & 29.9936 \\
\hline MSE & 1.0146 & 3.6138 & 6.8727 & 33.0220 & 65.1206 \\
\hline $\mathrm{NC}$ & 0.9844 & 0.9663 & 0.9443 & 0.8701 & 0.8301 \\
\hline The extracted watermark & & & & & \\
\hline
\end{tabular}

still distinguishable and perfect for all test images until the noise variance is set to 0.0005 . When the noise variance is set to 0.0005 and 0.001 , the extracted watermark is incomplete but distinguishable for all test images; the $\mathrm{NC}$ values are all above 0.8 . Therefore, the proposed algorithm can resist noise attack to some extent.

\subsubsection{The Comparison Experiments of Related Algorithm.}

To verify the effectiveness of the proposed algorithm, the proposed algorithm is compared with related algorithms based on DWT and DCT. DCT-based watermark algorithm is performed by embedding watermark in the largest $n \times n$ coefficients of the first-level DCT subband. Watermarking based on DWT is performed by embedding watermark in the largest $n \times n$ coefficients of the first-level subband, where a one-level DWT decomposition is performed using "Haar" wavelet.

To be fair, both the watermark image and the test images are the same to all algorithms. Table 7 shows the results of comparison on "Lena" image. As shown in Table 7, the proposed algorithm outperforms other algorithms in invisibility. Meanwhile, in robustness against these attacks, such as scaling attack and cropping attack, the proposed algorithm gets advantage over other algorithms.

In Table 2, although the NC values of proposed algorithm are not higher than other algorithms except no attack and scaling attack, it is noted that PSNR values of proposed algorithm are higher than other algorithms except cropping middle (50\%). In other words, watermark which is embedded in host image cause much degradation of perceptual quality of the watermarked image, yet the quality of the watermarked image for proposed algorithm is better.

\section{Conclusion}

This paper tries to implement an image watermark by taking advantage of the unique advantages of extended discrete shearlet and selecting watermark embedding location based on the highest information entropy. The unique advantages of extended discrete shearlet made the watermark system more invisible. Experimental results demonstrate that visual performance of the watermarked images is preferable to that of related algorithms based on DWT and DCT; meanwhile, the proposed watermark algorithm can resist common attack. Therefore, the extended discrete shearlet can be considered as an appropriate transform for digital watermark.

\section{Conflict of Interests}

The authors declare that there is no conflict of interests regarding the publication of this paper. 
TABLE 7: Comparison on "Lena” image (NC/PSNR).

\begin{tabular}{lccc}
\hline & DCT & DWT & DST \\
\hline No attack & $0.9849 / 34.7284 \mathrm{~dB}$ & $0.9849 / 33.7641 \mathrm{~dB}$ & $0.9849 / 53.1768 \mathrm{~dB}$ \\
Scaling (25\%) & $0.7699 / 27.8846 \mathrm{~dB}$ & $0.7686 / 28.0819 \mathrm{~dB}$ & $0.7778 / 29.9888 \mathrm{~dB}$ \\
Scaling (50\%) & $0.9243 / 31.6796 \mathrm{~dB}$ & $0.9261 / 31.6349 \mathrm{~dB}$ & $0.9302 / 34.6526 \mathrm{~dB}$ \\
Scaling (4) & $0.9809 / 34.7284 \mathrm{~dB}$ & $0.9749 / 33.8214 \mathrm{~dB}$ & $0.9819 / 45.9472 \mathrm{~dB}$ \\
JPEG (10) & $0.8428 / 29.2431 \mathrm{~dB}$ & $0.9824 / 29.0396 \mathrm{~dB}$ & $0.7056 / 30.6196 \mathrm{~dB}$ \\
JPEG (30) & $0.9429 / 31.9655 \mathrm{~dB}$ & $0.9844 / 31.5159 \mathrm{~dB}$ & $0.8252 / 35.1718 \mathrm{~dB}$ \\
JPEG (50) & $0.9819 / 32.5494 \mathrm{~dB}$ & $0.9849 / 32.0032 \mathrm{~dB}$ & $0.9004 / 36.4005 \mathrm{~dB}$ \\
Cropping right (50\%) & $0.7222 / 9.6540 \mathrm{~dB}$ & $0.9790 / 9.6459 \mathrm{~dB}$ & $0.9712 / 9.6617 \mathrm{~dB}$ \\
Cropping right (75\%) & $0.7280 / 7.0743 \mathrm{~dB}$ & $0.8530 / 7.0719 \mathrm{~dB}$ & $0.8579 / 7.0764 \mathrm{~dB}$ \\
Cropping middle (50\%) & $0.7100 / 11.6153 \mathrm{~dB}$ & $0.8750 / 27.3320 \mathrm{~dB}$ & $0.8901 / 11.6305 \mathrm{~dB}$ \\
Salt \& Pepper noise (0.1) & $0.6934 / 15.4339 \mathrm{~dB}$ & $0.6331 / 15.3887 \mathrm{~dB}$ & $0.6729 / 15.4918 \mathrm{~dB}$ \\
\hline
\end{tabular}

\section{Acknowledgments}

This work was supported by National Natural Science Foundation of China (no. 61379010) and Natural Science Basic Research Plan in Shaanxi Province of China (nos. 2012JQ1012 and 2015JM6293).

\section{References}

[1] G. Y. Liu, H. J. Liu, and A. Kadir, "Wavelet-based color pathological image watermark through dynamically adjusting the embedding intensity," Computational and Mathematical Methods in Medicine, vol. 2012, Article ID 406349, 10 pages, 2012.

[2] G. Bhatnagar, Q. M. J. Wu, and P. K. Atrey, "Secure randomized image watermarking based on singular value decomposition," ACM Transactions on Multimedia Computing, Communications and Applications, vol. 10, no. 1, article 4, 2013.

[3] Y. X. Ma, J. C. Xie, and J. G. Luo, "Image enhancement based on nonsubsampled contourlet transform," in Proceedings of the 5th International Conference on Information Assurance and Security (IAS '09), pp. 31-34, September 2009.

[4] H. Sadreazami and M. Amini, "A robust spread spectrum based image watermarking in ridgelet domain," AEU-International Journal of Electronics and Communications, vol. 66, no. 5, pp. 364-371, 2012.

[5] P. Mangaiyarkarasi and S. Arulselvi, "A new digital image watermarking based on finite ridgelet transform and extraction using lCA," in Proceedings of the International Conference on Emerging Trends in Electrical and Computer Technology, pp. 837-841, March 2011.

[6] G. Easley, D. Labate, and W.-Q. Lim, "Sparse directional image representations using the discrete shearlet transform," Applied and Computational Harmonic Analysis, vol. 25, no. 1, pp. 25-46, 2008.

[7] V. M. Patel, G. R. Easley, and J. Healy, "Shearlet-based deconvolution," IEEE Transactions on Image Processing, vol. 18, no. 12, pp. 2673-2685, 2009.

[8] S. Ranjbar, F. Zargari, and M. Ghanbari, "A highly robust twostage Contourlet-based digital image watermarking method," Signal Processing: Image Communication, vol. 28, no. 10, pp. 1526-1536, 2013.

[9] W.-Q. Lim, "The discrete shearlet transform: a new directional transform and compactly supported shearlet frames," IEEE
Transactions on Image Processing, vol. 19, no. 5, pp. 1166-1180, 2010.

[10] R. Gomathi and A. V. A. Kumar, "An efficient GEM model for image inpainting using a new directional sparse representation: Discrete Shearlet Transform," in Proceedings of the IEEE International Conference on Computational Intelligence and Computing Research (ICCIC '10), pp. 1-4, Coimbatore, India, December 2010.

[11] X.-Y. Wang, Y.-C. Liu, and H.-Y. Yang, "An efficient remote sensing image denoising method in extended discrete shearlet domain," Journal of Mathematical Imaging and Vision, vol. 49, no. 2, pp. 434-453, 2014.

[12] C. E. Shanonn and W. Weaver, The Mathematical Theory of Communication, University of Illinois Press, 1946.

[13] C. E. Shannon and E. Claude, "A mathematical theory of communication," Bell System Technical Journal, vol. 27, no. 3, pp. 379-423, 623-656, 1948.

[14] K. Guo and D. Labate, "Optimally sparse multidimensional representation using shearlets," SIAM Journal on Mathematical Analysis, vol. 39, no. 1, pp. 298-318, 2007. 


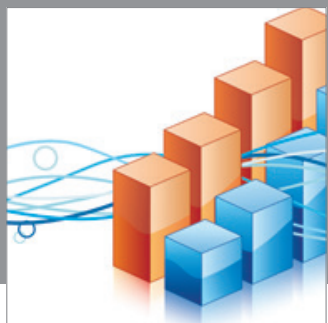

Advances in

Operations Research

mansans

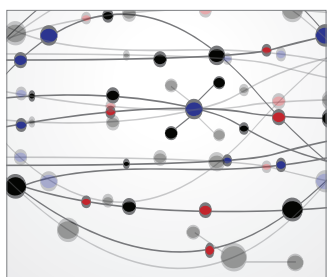

The Scientific World Journal
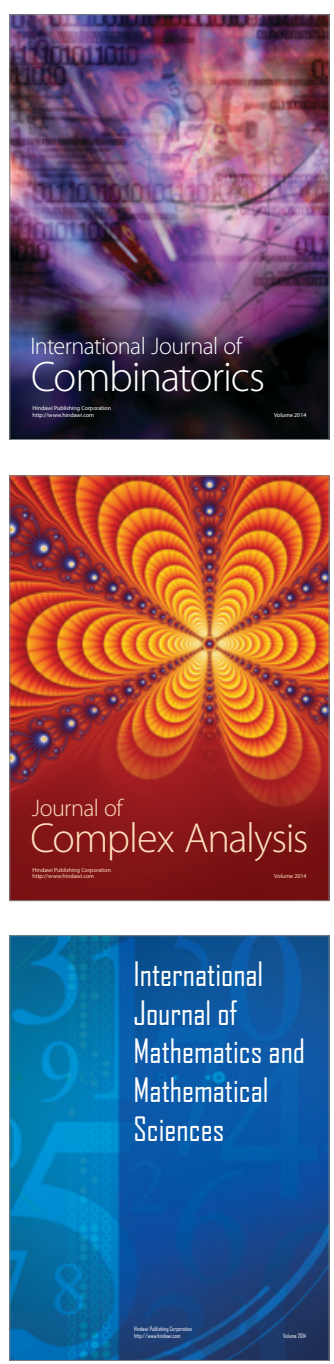
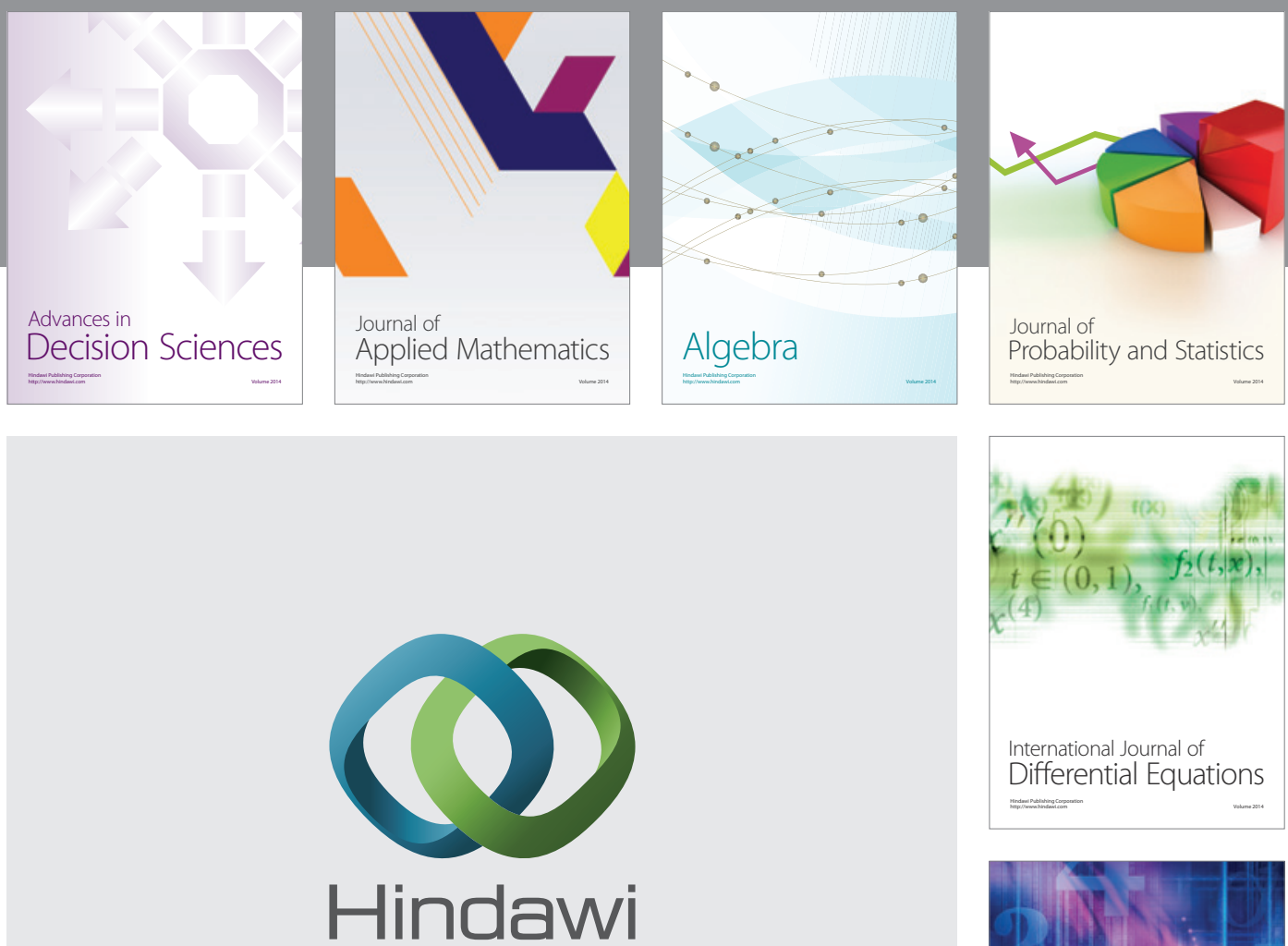

Submit your manuscripts at http://www.hindawi.com
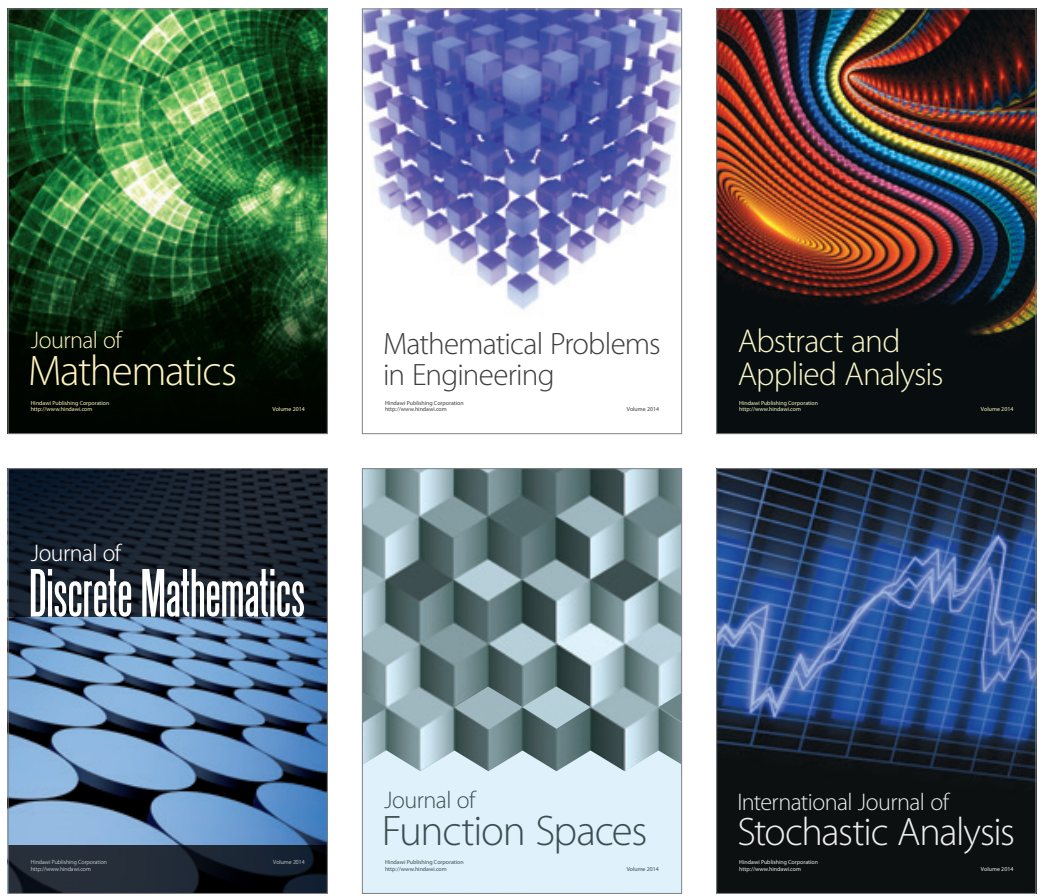

Journal of

Function Spaces

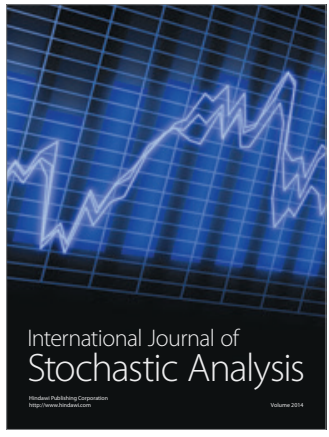

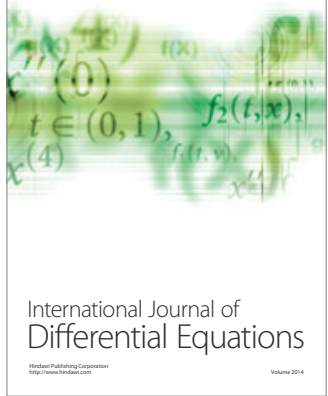
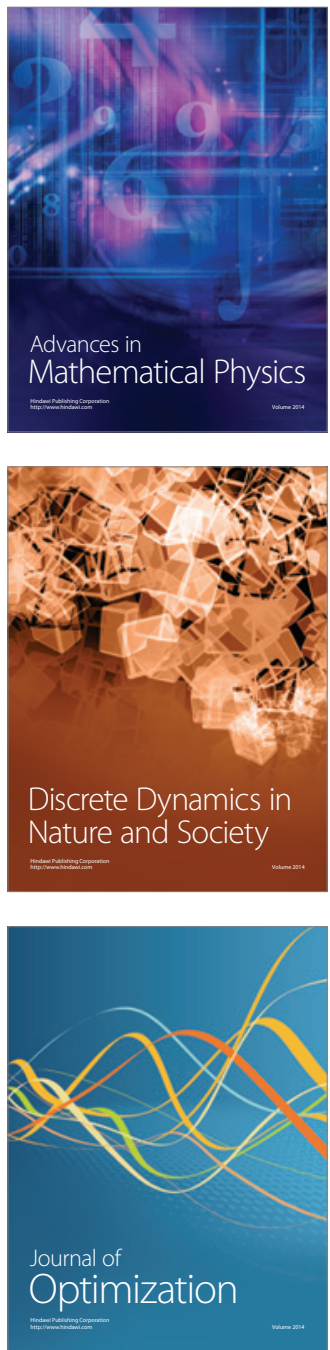